\title{
Subclinical myocardial dysfunction and reduced left ventricular global longitudinal strain in patients with COVID-19
}

\author{
Wei-Chieh Lee LiD $^{1,2}$
}

Received: 18 August 2021 / Accepted: 18 August 2021 / Published online: 21 August 2021

(c) The Author(s), under exclusive licence to Springer Nature B.V. 2021

The new severe acute respiratory syndrome coronavirus 2 is the etiological agent of novel coronavirus disease 2019 (COVID-19), which has caused a worldwide pandemic. COVID-19 can affect the cardiovascular system and cause acute myocarditis, various arrhythmias, and decompensated heart failure. A recently published article by Turan et al. [1] reported that COVID-19 causes subclinical myocardial dysfunction due to impaired left ventricular global longitudinal strain (LV-GLS) during the early recovery phase, even in low cardiac risk and asymptomatic COVID-19 patients. The author reported that the absolute values of LV-GLS were significantly lower in the COVID-19 group compared with the healthy control group $(19.17 \pm 2.65$ vs. $20.07 \pm 2.19$, respectively, $\mathrm{p}=0.03$ ), and the proportion of patients with impaired LV-GLS was significantly increased in the COVID19 group. Multivariable logistic regression analysis found that recovery from COVID-19 was a predictor for detecting impaired LV-GLS (absolute value $\leq 18$ ) (odds ratio, 0.133 $(0.038-0.461)$; $95 \% \mathrm{CI}, \mathrm{p}=0.001)$. In addition, right ventricular and diastolic function presented within the normal range and did not differ between the COVID-19 population and the healthy population. In this study, the COVID-19 population presented normal electrocardiography finding and normal cardiac marker values. Therefore, possible mechanisms of myocardial disruption occur at the tissue level or involve cardiomyocyte oxidative stress, and the impact exclusively influences LV-GLS.

This comment refers to the article available online at https://doi. org/10.1007/s 10554-021-02376-Z

\section{Wei-Chieh Lee}

leeweichieh@yahoo.com.tw

1 Institute of Clinical Medicine, College of Medicine, National Cheng Kung University, Tainan, Taiwan

2 Division of Cardiology, Department of Internal Medicine, Kaohsiung Chang Gung Memorial Hospital, Chang Gung University College of Medicine, No. 123, Ta Pei Road, Niao Sung District, Kaohsiung City 833, Taiwan
In COVID-19 infection, immune systems are activated by rapid viral replication and release cytokines and chemokines. The release of excessive proinflammatory cytokines and chemokines triggers inflammatory responses and leads to cytokine storms. The disease generally becomes fatal and causes multiorgan failure. The mechanism of myocardial injury in COVID-19 infection is unclear, but the involvement of the cardiovascular system is of high importance to predict morbidity and mortality. Myocardial injury can be explained by several potentially mechanisms, such as ischemic myocardial injury, myocarditis, microvascular damage, hypoxemia, arrhythmias, cytokine-related injury, or even stress cardiomyopathy [2].

LV-GLS is a sensitive measure of myocardial dysfunction, a powerful predictor ofoutcomes and more reproducible than left ventricular ejection fraction. LV-GLS was used to predict survival in patients with valvular heart disease, heart failure, and myocardial infarction. Recently, LV-GLS was applied as a monitoring method for patients with potential cardiotoxic agents. One previous study also noted reduced LV-GLS in hospitalized COVID-19 patients and showed no correlation between low strain and infection severity. Previous imaging studies also demonstrated the presence of cardiac sequelae on magnetic resonance imaging in hospitalized patients and outpatients with COVID19 infection. Therefore, COVID-19 infection is a cardiotoxic virus and affects the cardiovascular system, including the heart muscle of the $\mathrm{LV}$, regardless of the severity of infection.

Cardiac involvement predicts morbidity and mortality after COVID-19 infection, which may represent a direct cardiotoxic agent and involve the heart muscle of the LV. LV-GLS is an important method for the early identification of subclinical left ventricular myocardial dysfunction regardless of the severity of COVID-19 infection. 
Conflict of interest None.

Acknowledgements None.

Funding None.

\section{Declarations}

Conflict of interest The authors declare that they have no conflict of interest.

Disclosures None.

\section{References}

1. Turan T, Özderya A, Şahin S et al (2021) Left ventricular global longitudinal strain in low cardiac risk outpatients who recently recovered from coronavirus disease 2019. Int J Cardiovasc Imaging. https://doi.org/10.1007/s10554-021-02376-z

2. Magadum A, Kishore R (2020) Cardiovascular manifestations of COVID-19 infection. Cells 9(11):2508

Publisher's Note Springer Nature remains neutral with regard to jurisdictional claims in published maps and institutional affiliations. 